\title{
Persistence, Dose Titration, and Health Care Resource Utilization Among Crohn's Disease Patients Treated With Ustekinumab: A Real-World Analysis in the United States
}

\author{
Camilo Obando - Zhijie Ding · Erik Muser - Neel Vaidya • \\ Wenqin Qiang · Xiaoxi Sun · Huiqi Wang · Rajesh Mallampati • \\ Lin Xie
}

Received: December 11, 2019 / Published online: March 19, 2020

(c) The Author(s) 2020

\section{ABSTRACT}

Introduction: Crohn's disease (CD) is a chronic inflammatory disease of the gastrointestinal tract. This real-world study evaluated persistence, dose titration, health care resource utilization (HCRU) and associated costs, and medication use among CD patients treated with ustekinumab (UST) in several pooled US commercial database populations.

Methods: CD patients aged $\geq 18$ years with medical or pharmacy claims for UST were selected from pooled data from 3 large, national commercial databases. The first observed medical or pharmacy claim for UST was the index date. Patients were required to have had $\geq 1$ medical claim with a CD diagnosis during the

Enhanced Digital Features To view enhanced digital features for this article go to https://doi.org/10.6084/ m9.figshare.11876415.

Electronic Supplementary Material The online version of this article (https://doi.org/10.1007/s12325020-01276-3) contains supplementary material, which is available to authorized users.

C. Obando $\cdot$ Z. Ding $\cdot$ E. Muser $(\bowtie)$

Real World Value \& Evidence, Janssen Scientific

Affairs LLC, Horsham, PA, USA

e-mail: emuser@its.jnj.com

N. Vaidya · W. Qiang · X. Sun · H. Wang

R. Mallampati · L. Xie

Health Economics and Outcomes Research,

STATinMED Research, Ann Arbor, MI, USA
12 months prior to the index date and continuous health plan enrollment for a minimum of 12 months prior to and 12 months after the index date. Comparisons of outcomes during the baseline and follow-up periods were conducted using inferential statistical tests.

Results: A total of 214 eligible UST patients were selected. The majority $(74.8 \%)$ were biologic experienced (mean age: 41 years), and $83.6 \%$ remained treatment persistent during the 12 -month post-index period. Among discontinuers, $25.7 \%$ restarted UST, and $8.6 \%$ switched from UST in the 12-month observation period. The mean treatment duration was 329 days. Most patients (77\%) used the recommended UST dose, as defined as being within a $20 \%$ dose variation from label $(90 \mathrm{mg} /$ 8 weeks $\pm 20 \%), 17.9 \%$ experienced dose escalation, and $5.1 \%$ experienced dose reduction. Post-index immunomodulator and corticosteroid use reduced by $20 \%$ and $28 \%$, respectively, as compared with pre-index use among CD patients using UST. Annual all-cause ER visits and inpatient stays decreased by $20.5 \%$ and $30.3 \%$, respectively, with similar downward trends for annual CD-related HCRU.

Conclusions: The majority of CD patients prescribed UST were biologic experienced, and persistence was high over the 1-year follow-up. UST treatment initiation was associated with reductions in ER visits, inpatient stays, and steroid and other medication use. 
Keywords: Crohn's disease; Interleukin 12; Interleukin 23; Persistence; Resource utilization; Treatment patterns; Ustekinumab

\section{Key Summary Points}

Why carry out this study?

There is limited real-world evidence regarding the treatment patterns and health care utilization associated with ustekinumab (UST) among Crohn's disease $(\mathrm{CD})$ patients.

The current study evaluated persistence, dose titration, health care resource utilization (HCRU) and associated costs among CD patients treated with UST in the United States.

\section{What was learned from the study?}

The majority of CD patients prescribed UST were biologic experienced.

During the 12-month follow-up period, persistence to UST was $83.6 \%$. Among the $16.4 \%$ of patients who discontinued UST, $25.7 \%$ restarted UST, and $8.6 \%$ switched to another biologic.

The mean treatment duration was 329 days and the majority of patients used the labeled UST maintenance dose $(90 \mathrm{mg}$ every 8 weeks).

Post-UST initiation immunomodulator and corticosteroid use reduced by $20 \%$ and $28 \%$, respectively, whereas annual ER visits and inpatient stays decreased by $20.5 \%$ and $30.3 \%$, respectively, compared to pre-UST initiation.

\section{INTRODUCTION}

Crohn's disease (CD) is a chronic inflammatory gastrointestinal disorder that may affect any part of the digestive tract $[1,2]$. Presenting symptoms vary and can include abdominal pain, diarrhea, weight loss, depression, and perianal fistula [1, 3]. Approximately 780,000 Americans had CD in 2014, with increasing prevalence $[4,5]$. The burden of illness associated with $\mathrm{CD}$ is substantial, with extended hospital stays and frequent emergency department visits, and almost $80 \%$ of patients with CD require hospitalization at some point during their illness $[1,6,7]$. Health-related quality of life is consistently lower among CD patients in comparison with population norms [8].

Treatment strategies, which include surgery and medical options, are based on the severity and types of complications within the anatomic area affected by CD [9]. The American College of Gastroenterology (ACG) recommends treatment with biologic agents such as adalimumab (ADA), certolizumab pegol (CZP), infliximab (IFX), and vedolizumab (VDZ) for patients who fail to respond, lose response, or are intolerant to conventional treatment $[1,10,11]$. These treatments are prescribed to attain and maintain clinical remission, slow disease progression, and delay surgical interventions [12]. However, between one-third and one-half of patients do not respond to anti-tumor necrosis factor (antiTNF) treatments, which necessitates the introduction of a second biologic. Evidence shows a loss of response or intolerance occurs in almost $50 \%$ of patients treated with any biologic [13-17]. Loss of response to biologics generally leads to dose escalation, occurring in $17-35 \%$ of patients with $\mathrm{CD}$, with dose escalation rates ranging from $32 \%$ to $38 \%[18,19]$. Treatment discontinuation is also common and is observed in $15 \%$ of patients treated with IFX, and $21.7 \%$ of patients treated with CZP. Switching to other medications ranges from $11 \%$ to $35 \%$ [20]. Brady et al. [12] noted that only one-third of CD patients remained on a biologic treatment over a 12-month follow-up period.

As disease progression is noted in $70-80 \%$ of patients with $\mathrm{CD}$, there is a need for therapies that achieve and sustain adequate control of bowel inflammation, as defined by Lichtenstein et al. [1]. In clinical trials conducted among CD patients, ustekinumab (UST), an antibody to the p40 subunit of interleukin (IL)-12 and IL-23 [21], has significantly increased rates of clinical remission, clinical response, and corticosteroid- 
free clinical remission as compared with placebo [22]. As of 2018, the ACG guidelines have recommended the use of UST for patients with moderate-to-severe CD who failed previous treatment with corticosteroids, thiopurines, methotrexate, or anti-TNF inhibitors, or who have had no prior exposure to anti-TNF inhibitors. UST administration has also been found to reduce CD-related hospitalizations, surgeries, and alternative biologic therapies $[1,23]$.

However, real-world evidence in this area is limited. Such data can provide a better understanding of the performance of UST among CD patients with a 12-month follow-up in the realworld setting. Hence, the current study was conducted among CD patients treated with UST, to evaluate persistence, dose titration, health care resource utilization (HCRU) and associated costs, medications for treating $\mathrm{CD}$, and medications for treating CD-related symptoms or comorbid conditions.

\section{METHODS}

\section{Data Sources}

This retrospective observational study used data pooled from the IBM $^{\circledR}$ MarketScan ${ }^{\circledR}$ (IBM; September 26, 2015-December 31, 2017) as well as the Optum Clinformatics ${ }^{\mathrm{TM}}$ Data Mart (Optum) and IQVIA PharMetrics Plus ${ }^{\text {TM }}$ (IQVIA; September 26, 2015-March 31, 2018) databases. Different study periods were used due to different data availability from each database. All databases used were independent and contained no personal identifiable information. Because it is possible for individual patients to be included in more than 1 database, probabilistic matching along with an algorithm incorporating age, sex, hospital admission date, hospital discharge date, and primary discharge diagnosis were used to identify potential duplicates among similar patient records from multiple data sources $[24,25]$. In this sample, 5 (2.3\%) patients were identified with similar information. As the datasets were de-identified, it could not be determined with certainty if they were the same patients or patients with similar information. However, this potential overlap between datasets was below the predetermined $3 \%$ threshold [26], so these patients were included in the analysis.

\section{Sample Selection}

The US Food and Drug Administration approved UST on September 23, 2016, for the treatment of moderately-to-severely active CD, and the following Monday (September 26, 2016) was used as the start of the patient selection period. To allow a 12-month baseline period, patients who had at least 1 medical or pharmacy claim for UST between September 26, 2016 and March 31, 2018 (Optum and IQVIA) and between September 26, 2016 and December 31, 2017 (IBM) were included in the study. The index date was the date of the first medical or pharmacy claim for UST during the identification period; medical claims were identified by specified Healthcare Common Procedure Coding System (HCPCS) codes C9487, Q9989, J3358, and J3357, and pharmacy claims were identified by national drug code (NDC) data. Patients were required to have at least $1 \mathrm{CD}$ claim-based diagnosis [International Classification of Disease, Ninth Revision, Clinical Modification (ICD-9-CM) code 555.xx and ICD-10$\mathrm{CM}$ code K50.xx] during the 12 months pre-index date. Patients were aged at least 18 years on the index date and had at least 12 months of continuous health plan enrollment with medical and pharmacy benefits prior to (baseline period) and after (follow-up period) the index date.

Confirmed ulcerative colitis (UC) patients and patients with an equal number of diagnoses of CD and UC were excluded from the analysis $[17,27,28]$. This is important because the distinction between CD and UC can sometimes be challenging. As both UC and $\mathrm{CD}$ can have pathological features that overlap, pathologists sometimes choose to render a diagnosis of indeterminate colitis $[29,30]$. Hence, to identify true CD patients, an algorithm was used to derive classification (Supplemental Fig. 1). Patients were classified as UC or CD based on the majority number of inpatient claims. If there were equal inpatient claims for both 
diseases, the majority number of outpatient claims were evaluated. Finally, if outpatient claims for UC and CD were also equal, the patients were excluded from the study.

Patients with diagnostic codes for psoriasis (PsO), psoriatic arthritis, rheumatoid arthritis, ankylosing spondylitis, relapsing polychondritis, plaque $\mathrm{PsO}$, or pregnancy during the study period were excluded. Patients were also excluded if they had claims for multiple biologic agents on the index date [multiple occurrences of ADA, CZP, IFX, natalizumab (NTZ), UST, or VDZ], or claims for UST within 12 months before the index date.

Baseline characteristics, such as age, sex, US geographic region, health plan, and individual comorbidities were examined prior to the index date.

\section{Outcome Measures}

\section{Persistence}

Persistence was defined as the continuation of treatment with UST with an allowable gap of 90 days over a 12-month period.

\section{Discontinuation}

Discontinuation was defined as the absence of an additional UST claim within 90 days after the run-out date (last day of supply of the previous UST administration) or a switch to a non-index biologic. The discontinuation date was defined as the run-out date of the last UST claim, except when a switch (defined below) occurred before the run-out date, in which case the switch date was the discontinuation date.

Among discontinuers, patients who restarted UST after the 90-day period following the runout date of the index biologic were designated as restarters. The restart date was defined as the date the patient restarted UST. Patients who had a claim of a non-index biologic before the runout date of the index biologic or within 90 days after the discontinuation date were considered switchers. The switch date was defined as the date the patients initiated a non-index biologic. UST patients who did not have a claim for any biologics (ADA, CZP, IFX, NTZ, VDZ) after the 90 days following the run-out date were designated as discontinued without restart/switch. Duration of treatment during the 12 months follow-up was defined as the time from the index date to discontinuation (or switch date if a switch occurred first) for patients who discontinued treatment, and from the index date to study end for patients who remained on treatment.

\section{Dose Titration}

Among those who had 2 or more maintenance doses in the follow-up period, the overall average maintenance dose per 8 weeks (including $\pm 20 \%$ variation around the labeled maintenance dose of $90 \mathrm{mg}$ Q8W subcutaneously) was calculated for each patient, starting with the first maintenance dose, and censoring at the earliest of discontinuation or end of follow-up. The start of the maintenance dose was defined as the earliest evidence of subcutaneous ustekinumab. Patients who did not have any subcutaneous UST were excluded from any dose titration calculations. Doses above this 20\% variation from the United States Food and Drug Administration (FDA)-labeled dosing threshold were designated as dose escalation (>108 mg/8 weeks), while doses below the 20\% variation threshold were considered dose reduced $(<72 \mathrm{mg} / 8$ weeks). Sensitivity analyses were conducted for dosing variations by applying thresholds of $30 \%$ (dose escalation: $>117 \mathrm{mg}$ / 8 weeks; dose reduction: $<63 \mathrm{mg} / 8$ weeks), and $50 \%$ (dose escalation: $>135 \mathrm{mg} / 8$ weeks; dose reduction: $<45 \mathrm{mg} / 8$ weeks). Additionally, proportions were evaluated for patients (using $0 \%$ variation) who had $50 \%$ dose reduction $(\leq 45 \mathrm{mg} /$ 8 weeks), $50 \%$ dose escalation $(\geq 135 \mathrm{mg} /$ 8 weeks), and $100 \%$ dose escalation $(\geq 180 \mathrm{mg} /$ 8 weeks).

\section{Medication Adherence}

Medication Possession Ratio (MPR) MPR was calculated for patients with UST use, defined as the sum of days of supply for UST divided by the total number of days in a 12-month follow-up period. Patients with MPR $\geq 80 \%$ were considered adherent. This measure may over-estimate adherence, as overlapping prescription fill dates are not accounted for in the calculation (i.e., they are double-counted). While over- 
estimation of adherence is a possibility, prescription fills and injections may not occur on the same date, thus subtracting these overlapping days might result in under-estimation of adherence.

Proportion of Days Covered (PDC) PDC was defined as the number of days in the study period covered by the index medication prescription, divided by the total number of days in the 12-month follow-up period. Patients with PDC $\geq 80 \%$ were considered adherent. Since the numerator in this equation consisted of days covered by the biologic, the calculation did not double-count any overlap between prescription fill dates if, for example, a patient received an early refill of their medication. Hence, this estimate is a more conservative method of defining adherence, as compared with MPR.

\section{Medications for Treating CD}

The proportion of patients who had at least one claim for immunomodulators, antibiotics, 5 -aminosalicylates, or corticosteroids in the preindex period was compared with the proportion of patients who had at least one claim for the same medications during the post-index period $[31,32]$. Additionally, patients who used corticosteroids continuously for $\geq 3$ months (allowing for a 15-day gap between two claims) were also compared in the pre- and post-index periods.

\section{Medications for CD-Related Symptoms or Comorbid Conditions}

The proportion of patients who used medications for CD-related symptoms or for select comorbid conditions among CD patients was assessed and compared during the baseline and follow-up periods, within the following drug classes: opioid pain medications, anxiolytics, antidepressants, antispasmodics, and anti-diarrheal drugs [33].

\section{Health Care Resource Utilization and Associated Costs}

All-cause and CD-related emergency room visits, hospitalizations, CD-related surgeries and their associated costs were examined and compared for the baseline and follow-up periods.

\section{Statistical Methods}

Descriptive analyses were conducted separately for the 3 data sources prior to analyses on the pooled patient population. Variables included patient demographics, US geographic region, health plan type, and baseline comorbidities. Medications for CD treatment, management of CD-related symptoms or comorbid conditions, HCRU and associated costs were compared before and after the index date. All-cause as well as CD-related HCRU and associated costs, medications for treating $\mathrm{CD}$, and medications for CD-related symptoms or comorbid conditions were compared between the 12 -month baseline period and the 12-month follow-up period, using a bivariate comparison with a paired two-sample $t$ test (for continuous variables) or McNemar's test (for categorical variables). All statistical analyses were performed using the Statistical Analysis System (SAS) v.9.4 (Cary, NC, USA).

\section{Compliance with Ethics Guidelines}

The study did not involve the collection, use, or transmittal of individual identifiable data, and instead relied solely on the secondary use of depersonalized data; hence, institutional review board approval was not required. Secondary use of depersonalized data is explicitly exempted from ethics review per the "Common Rule": 45 CFR 46; Exemption 4); Federal Policy for the Protection of Human Subjects (1991), and the depersonalized data are not covered by the Privacy Rule, 45 CFR § 164.514 (b)(2)(i); Health Insurance Portability and Accountability Act of 1996 (HIPAA).

\section{RESULTS}

Based on study selection criteria, 214 CD patients on UST were included (Optum: 67; IQVIA: 73; IBM: 74; Fig. 1). On the index date $78.0 \%(n=167)$ patients had a medical or 
pharmacy claim for subcutaneous UST, whereas $22.0 \%(n=47)$ patients had a medical claim for intravenous (IV) UST.

\section{Baseline Characteristics}

The descriptive baseline characteristics of the pooled database are shown in Table 1 . The mean age of patients was 41 years, with the majority of patients aged between 18 and 54 years $(81.8 \%)$. Males constituted $57.9 \%$ of the sample. A large proportion of patients were from the southern region (36.0\%) of the United States. Most patients were commercially insured (96.7\%). The 3 most common comorbidities were anemia $(44.4 \%)$, depression $(23.4 \%)$, and anxiety (22.9\%). CD patient claims were associated with significant use of CD-related medications during the 12-month baseline period, specifically antibiotics (79.0\%), corticosteroids (57.5\%), and opioid pain medications (54.2\%). Of the $214 \mathrm{CD}$ patients included in the study, $74.8 \% \quad(n=160)$ were biologic experienced (Optum: 61.2\%; IQVIA: 79.5\%; and IBM: 82.4\%; Fig. 1$) ; 59.3 \%(n=127)$ had failed 1 biologic (ADA, IFX, CZP, or VDZ); and $15.4 \%(n=33)$ had failed 2 biologics. Most baseline characteristics were similar across all databases, except the proportion of patients with depression, anxiety, and hypertension. Optum had a numerically lower proportion of patients with depression (Optum: 16.4\%; IQVIA: 24.7\%; IBM: 28.4\%); anxiety (Optum: 14.9\%; IQVIA: 25.7\%; IBM: 27.4\%); and hypertension (Optum: 14.9\%; IQVIA: $20.6 \%$; IBM: $21.6 \%$ ), as compared with other databases.

\section{Outcome Measures}

\section{Persistence}

Based on a 90-day allowable gap period, 179 $(83.6 \%)$ patients treated with UST were persistent with treatment at 12 months of follow-up (Table 2). Among the 35 (16.4\%) patients who discontinued UST treatment, 9 (25.7\%) restarted UST after the 90-day allowable discontinuation gap, and $3(8.6 \%)$ switched to another biologic before or within 90 days after the discontinuation date (Table 2). The remaining 23
(65.7\%) patients who discontinued UST treatment did not restart or switch to other biologics within the 12-month follow-up period. The average time to discontinuation among patients who discontinued UST was 138 days (Table 2). The mean duration of therapy among all CD patients treated with UST during 12 months of follow-up was 329 days.

\section{Adherence}

Average MPR was 0.79 , and more than $50 \%$ of the patients were adherent $(\mathrm{MPR} \geq 0.80)$ to their treatment by this definition. Average PDC was found to be 0.73 (overall adherence was consistent across the 2 definitions).

\section{Maintenance Dose Titration}

There were 196 patients who had $\geq 2$ maintenance doses in the pooled sample and were included in the dose titration analysis. Dose titration results are shown in Table 3. The majority of these patients (77\%) utilized FDAlabeled doses (using a 20\% variation threshold), 35 (17.9\%) patients experienced dose escalation (> $108 \mathrm{mg} / 8$ weeks), and $10(5.1 \%)$ patients experienced dose reduction $(<72 \mathrm{mg} / 8$ weeks). The percentage of dose titration reduced as the variation window increased. Without applying any variation (0\%) threshold, $15(7.7 \%)$ patients experienced $50 \%$ dose escalation $(\geq 135 \mathrm{mg} /$ 8 weeks), 6 (3.1\%) experienced 100\% dose escalation ( $\geq 180 \mathrm{mg} / 8$ weeks), and no patients (0\%) experienced $50 \%$ dose reduction ( $\leq 45 \mathrm{mg} / 8$ weeks), as compared with the label dose $(90 \mathrm{mg} / 8$ weeks) during the follow-up period. When a $30 \%$ variation threshold was applied to each dosing intensity window, 27 (13.8\%) patients experienced dose escalation (> $117 \mathrm{mg} / 8$ weeks), and 2 (1\%) patients experienced dose reduction $(<63 \mathrm{mg} / 8$ weeks).

\section{Baseline Versus Follow-Up Analysis} of Medications for Treating CD, Medications for CD-Related Symptoms or Comorbid Conditions, Health Care Resource Utilization, and Associated Costs

In the 12-month baseline versus the 12-month follow-up period, decreases were noted in the proportion of patients using medications for 


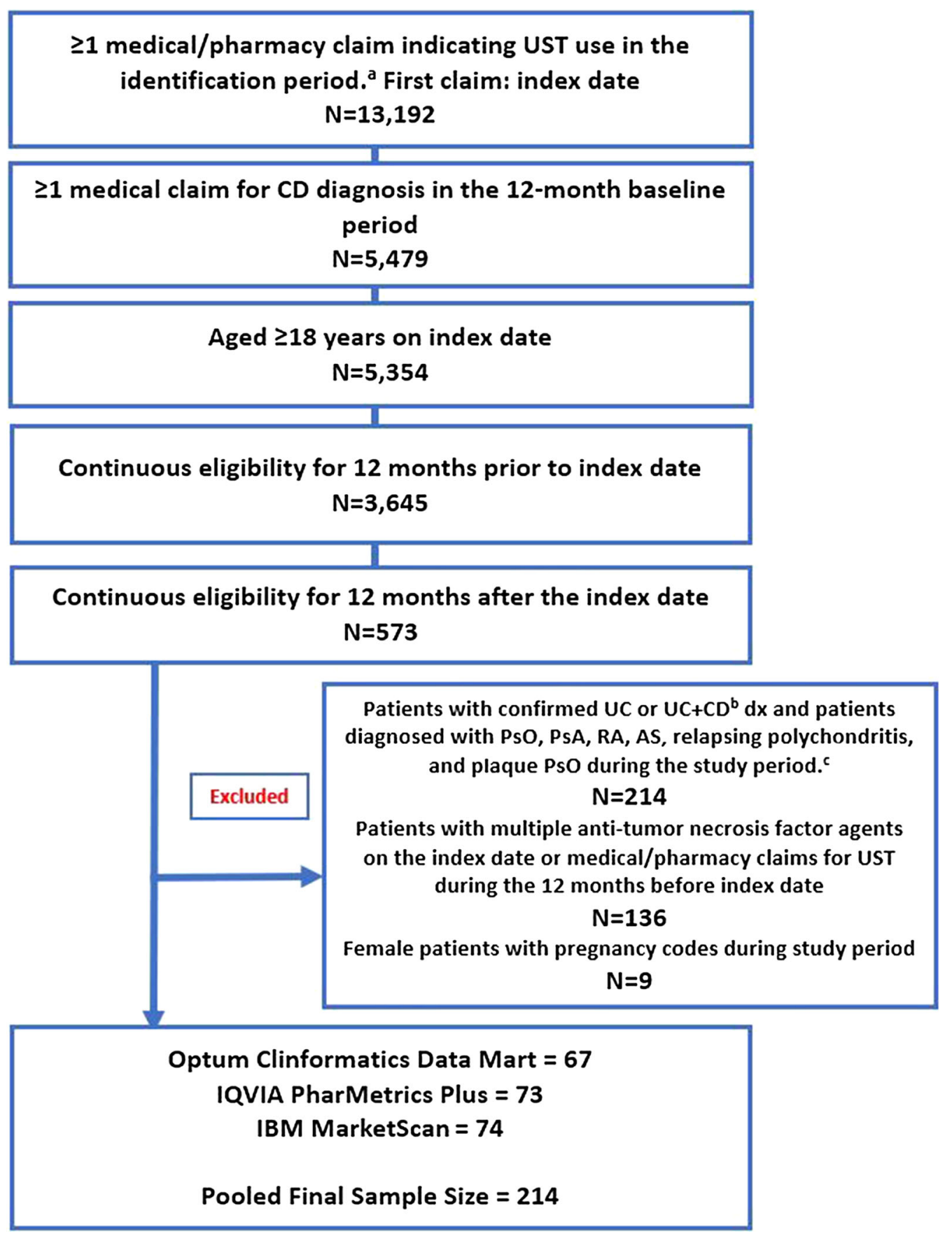

Fig. 1 Sample selection. $A S$ ankylosing spondylitis; $C D$ Crohn's disease; $P s O$ psoriasis, $P s A$ psoriatic arthritis; $R A$ rheumatoid arthritis; UST ustekinumab; $U C$, ulcerative colitis. ${ }^{a}$ Identification period: IQVIA PharMetrics Plus ${ }^{\text {TM }}$ and Optum Clinformatics ${ }^{\mathrm{TM}}$ Data Mart: 09/26/ 2016-03/31/2018; $\quad$ IBM $^{\circledR} \quad$ MarketScan ${ }^{\circledR}$ : 09/26/ 2016-12/31/2017. b $(\mathrm{dx}=$ diagnosis $)$. Using the algorithm (details in the methods section), patients with confirmed UC (number of inpatient/outpatient claims of $\mathrm{UC}>\mathrm{CD}$ ) and confirmed UC + CD (both inpatient/ outpatient claims of UC $=\mathrm{CD}$ ) diagnosis were excluded,

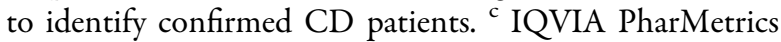
Plus ${ }^{\text {TM }}$ and Optum Clinformatics ${ }^{\text {TM }}$ Data Mart: 09/26/ 2015- 03/31/2018; $\quad$ IBM $^{\circledR}$ : MarketScan $^{\circledR}$ : 09/26/ 2015-12/31/2017 
Table 1 Descriptive baseline characteristics

\begin{tabular}{|c|c|c|}
\hline & \multicolumn{2}{|c|}{$\begin{array}{l}\text { Pooled } \\
\text { population } \\
n=214\end{array}$} \\
\hline & $\begin{array}{l}n / \\
\text { Mean }\end{array}$ & $\begin{array}{l}\% / \\
\text { SD }\end{array}$ \\
\hline Age & 40.6 & 14.0 \\
\hline \multicolumn{3}{|l|}{ Age group (years) } \\
\hline $18-34$ & 76 & $35.5 \%$ \\
\hline $35-54$ & 99 & $46.3 \%$ \\
\hline $55-64$ & 30 & $14.0 \%$ \\
\hline$\geq 65$ & 9 & $4.2 \%$ \\
\hline \multicolumn{3}{|l|}{ Sex } \\
\hline Male & 124 & $57.9 \%$ \\
\hline Female & 90 & $42.1 \%$ \\
\hline \multicolumn{3}{|l|}{ US geographic region } \\
\hline Northeast & 50 & $23.4 \%$ \\
\hline North Central & 50 & $23.4 \%$ \\
\hline South & 77 & $36.0 \%$ \\
\hline West & 37 & $17.3 \%$ \\
\hline \multicolumn{3}{|l|}{ Plan type } \\
\hline $\begin{array}{l}\text { Commercial (includes self-insured for } \\
\text { IQVIA) }\end{array}$ & 207 & $96.7 \%$ \\
\hline Medicare & 5 & $2.3 \%$ \\
\hline Other/missing & 2 & $0.9 \%$ \\
\hline \multicolumn{3}{|l|}{ Comorbidities } \\
\hline Anemia & 95 & $44.4 \%$ \\
\hline Depression & 50 & $23.4 \%$ \\
\hline Anxiety & 49 & $22.9 \%$ \\
\hline Hypertension & 41 & $19.2 \%$ \\
\hline Chronic pulmonary disease & 25 & $11.7 \%$ \\
\hline Mild liver disease & 23 & $10.7 \%$ \\
\hline Obesity & 14 & $6.5 \%$ \\
\hline Peptic ulcer disease & 10 & $4.7 \%$ \\
\hline Peripheral vascular disease & 9 & $4.2 \%$ \\
\hline Venous thromboembolism & 9 & $4.2 \%$ \\
\hline
\end{tabular}

Table 1 continued

\begin{tabular}{|c|c|c|}
\hline & \multicolumn{2}{|c|}{$\begin{array}{l}\text { Pooled } \\
\text { population } \\
n=214\end{array}$} \\
\hline & $\begin{array}{l}n / \\
\text { Mean }\end{array}$ & $\begin{array}{l}\% / \\
\text { SD }\end{array}$ \\
\hline Cholelithiasis & 9 & $4.2 \%$ \\
\hline $\begin{array}{l}\text { Any malignancy, except malignant } \\
\text { neoplasm of skin }\end{array}$ & 8 & $3.7 \%$ \\
\hline Diabetes without chronic complication & 7 & $3.3 \%$ \\
\hline Diabetes mellitus & 7 & $3.3 \%$ \\
\hline Renal disease & 5 & $2.3 \%$ \\
\hline Myocardial infarction/atherosclerosis & 6 & $2.8 \%$ \\
\hline Congestive heart failure & 2 & $0.9 \%$ \\
\hline AIDS/HIV & 2 & $0.9 \%$ \\
\hline Cerebrovascular disease & 1 & $0.5 \%$ \\
\hline Moderate or severe liver disease & 1 & $0.5 \%$ \\
\hline
\end{tabular}

AIDS acquired immunodeficiency syndrome; CD Crohn's disease; $H I V$ human immunodeficiency virus; $S D$ standard deviation; UST ustekinumab

treating CD. A 20.0\% decrease in the proportion of patients using immunomodulators was noted from baseline (BL) to follow-up (FU) (BL: 46.7\% vs. FU: $37.4 \% ; p=0.005$; Fig. 2 ). The proportion of patients utilizing 5-aminosalicylates decreased by $47.4 \%$ (BL: $28.5 \%$ vs. FU: $15.0 \%$; $p<0.001)$, and the proportion using corticosteroids declined by $28.0 \%$ (BL: $79.9 \%$ vs. FU: $57.5 \% ; p<0.001$; Fig. 2) during follow-up as compared with the baseline period. The proportion of patients who used corticosteroids for $\geq 3$ continuous months numerically declined by $23.9 \%$ (BL: $11.7 \%$ vs. FU: $8.9 \%$; $p=0.304$ ), but was not statistically significant. The proportion of patients using anxiolytics (BL: $29.0 \%$ vs. FU: $22.4 \% ; p=0.039$ ) and antispasmodics (BL: $23.4 \%$ vs. FU: $17.3 \%$; $p=0.024)$ decreased significantly during followup, by $22.8 \%$ and $26.1 \%$, respectively (Fig. 3).

The baseline versus follow-up results for allcause and CD-related ER visits, hospitalizations, and CD-related surgeries and associated costs 
Table 2 Treatment patterns

\begin{tabular}{llc}
\hline & Pooled population & \\
\cline { 2 - 3 } & $\boldsymbol{n}=\mathbf{2 1 4}$ & \%/SD \\
\hline Persistence & 179 & $83.6 \%$ \\
Discontinuation & 35 & $16.4 \%$ \\
$\quad$ Time to discontinuation, days (among & 137.7 & 76.1 \\
$\quad$ discontinued patients) & & $25.7 \%$ \\
Re-start of index biological & 9 & 79.0 \\
Time to restart of UST, days & 206.0 & $8.6 \%$ \\
Switched from UST & 3 & 63.0 \\
Time to switch, days & 172.7 & $65.7 \%$ \\
Discontinued without restart or switch & 23 & 73.5 \\
Time to discontinuation without & 152.2 & 89.9 \\
$\quad$ restart or switch, days & & \\
Duration of therapy among all patients & 328.7 & 0.30 \\
Medication adherence & & $52.3 \%$ \\
Medication possession ratio (MPR) & 0.79 & 0.25 \\
MPR $\geq 80 \%$ & 112 & $48.1 \%$ \\
Proportion of days covered (PDC) & 0.73 & \\
PDC $\geq 80 \%$ & 103 & \\
\hline PD & &
\end{tabular}

$C D$ Crohn's disease; $S D$ standard deviation; UST ustekinumab

are shown in Table 4. Emergency department/room (ER) visits and hospitalizations are reported among those who experienced an event. Results show a $20.5 \%$ reduction in the proportion of patients with all-cause ER visits (BL: $47.7 \%$ vs. FU: $37.9 \% ; p=0.013$ ) and a $30.3 \%$ reduction in the proportion of patients with inpatient stays (BL: $41.6 \%$ vs. FU: $29.0 \%$; $p=0.004$ ) from baseline to follow-up. CD-related ER visits decreased during follow-up by $43.4 \%$ (BL: $34.6 \%$ vs. FU: $19.6 \% ; p<0.001$ ) and CD-related inpatient stays decreased by $31.2 \%$ (BL: $40.7 \%$ vs. FU: $28.0 \% ; p=0.004$ ). During follow-up, the average number of CD-related ER visits (BL: 0.6 vs. FU: $0.4 ; p=0.018$ ) and $\mathrm{CD}$ surgeries (BL: 1.4 vs. FU: $1.0 ; p=0.010$ ) also declined by $33.3 \%$ and $28.6 \%$, respectively. ER costs during follow-up decreased by $\$ 809$ for allcause utilization (BL: $\$ 2182$ vs. FU: \$1373; $p=0.013)$ and by $\$ 815$ for CD-related utilization (BL: $\$ 1293$ vs. FU: $\$ 478 ; p=0.002$ ). Inpatient costs showed a non-significant numeric reduction of $\$ 1686$ for all-cause (BL: $\$ 14,054$ vs. FU: $\$ 12,368 ; p=0.500)$ and a numeric reduction of $\$ 1,828$ for CD-related (BL: $\$ 12,701$ vs. FU: $\$ 10,873 ; p=0.457)$ utilization during the follow-up as compared with the baseline period.

\section{DISCUSSION}

All clinical trials with UST, as compared with placebo, have indicated superiority in clinical response and HCRU reduction for both the induction and maintenance phases of 
Table 3 Dose titration

\begin{tabular}{|c|c|c|c|c|}
\hline Dose titration & & $n$ & $\%$ & Cumulative \% \\
\hline \multicolumn{5}{|l|}{$0 \%$ variation } \\
\hline$\leq 45 \mathrm{mg} / 8$ weeks & $50 \%$ Dose reduction & 0 & $0.0 \%$ & \\
\hline$\geq 135 \mathrm{mg} / 8$ weeks & $50 \%$ Dose escalation & 15 & $7.7 \%$ & \\
\hline$\geq 180 \mathrm{mg} / 8$ weeks & $100 \%$ Dose escalation & 6 & $3.1 \%$ & \\
\hline \multicolumn{5}{|l|}{$20 \%$ variation } \\
\hline$\geq 144 \mathrm{mg} / 8$ weeks & Dose escalation & 13 & $6.6 \%$ & $6.6 \%$ \\
\hline$(108-144) \mathrm{mg} / 8$ weeks & Dose escalation & 22 & $11.2 \%$ & $17.9 \%$ \\
\hline [72-108] mg/8 week-label & Label & 151 & $77.0 \%$ & $94.9 \%$ \\
\hline$(48-72) \mathrm{mg} / 8$ weeks & Dose reduction & 10 & $5.1 \%$ & $100.0 \%$ \\
\hline$\leq 48 \mathrm{mg} / 8$ weeks & Dose reduction & 0 & $0.0 \%$ & $100.0 \%$ \\
\hline \multicolumn{5}{|l|}{$30 \%$ variation } \\
\hline$\geq 156 \mathrm{mg} / 8$ weeks & Dose escalation & 10 & $5.1 \%$ & $5.1 \%$ \\
\hline$(117-156) \mathrm{mg} / 8$ weeks & Dose escalation & 17 & $8.7 \%$ & $13.8 \%$ \\
\hline [63-117] mg/ 8 week-label & Label & 167 & $85.2 \%$ & $99.0 \%$ \\
\hline$(40-63) \mathrm{mg} / 8$ weeks & Dose reduction & 2 & $1.0 \%$ & $100.0 \%$ \\
\hline$\leq 40 \mathrm{mg} / 8$ weeks & Dose reduction & 0 & $0.0 \%$ & $100.0 \%$ \\
\hline \multicolumn{5}{|l|}{$50 \%$ variation } \\
\hline$\geq 180 \mathrm{mg} / 8$ weeks & Dose escalation & 6 & $3.1 \%$ & $3.1 \%$ \\
\hline$(135-180) \mathrm{mg} / 8$ weeks & Dose escalation & 9 & $4.6 \%$ & $7.7 \%$ \\
\hline [45-135] mg/8 week-label & Label & 181 & $92.3 \%$ & $100.0 \%$ \\
\hline$<45 \mathrm{mg} / 8$ weeks & Dose reduction & 0 & $0.0 \%$ & $100.0 \%$ \\
\hline
\end{tabular}

CD Crohn's disease; UST ustekinumab

treatment among CD patients who previously failed or were intolerant to TNF antagonists and conventional therapies $[22,27,28]$. This retrospective database analysis is one of the first realworld studies to examine the outcomes of UST treatment among CD patients in the United States. Leveraging pooled data from 3 large US commercial claims databases, this study demonstrated that, among CD patients who initiated UST, $74.8 \%$ were biologic experienced, $83.6 \%$ remained persistent to their therapy, and $77 \%$ used the FDA-recommended doses (i.e., did not require dose titration) 1 year after the index date. Moreover, reductions in ER visits, inpatient stays, corticosteroid, and immunomodulator use were observed after patients initiated UST treatment.

ACG guidelines recommend UST treatment for moderate-to-severe $\mathrm{CD}$ patients who have failed previous treatment with corticosteroids, thiopurines, methotrexate, or anti-TNF inhibitors, or who have had no prior exposure to antiTNF inhibitors. Persistence to biologic treatment is generally used as a proxy for the risk-benefit profile of the treatment. A realworld study of TNF-inhibitors (ADA, CZP, IFX) reported that $38-45 \%$ of $\mathrm{CD}$ patients discontinued their anti-TNF therapy within 12 months [19]. Another recently published real-world study reported that only $48.5 \%$ of the CD 


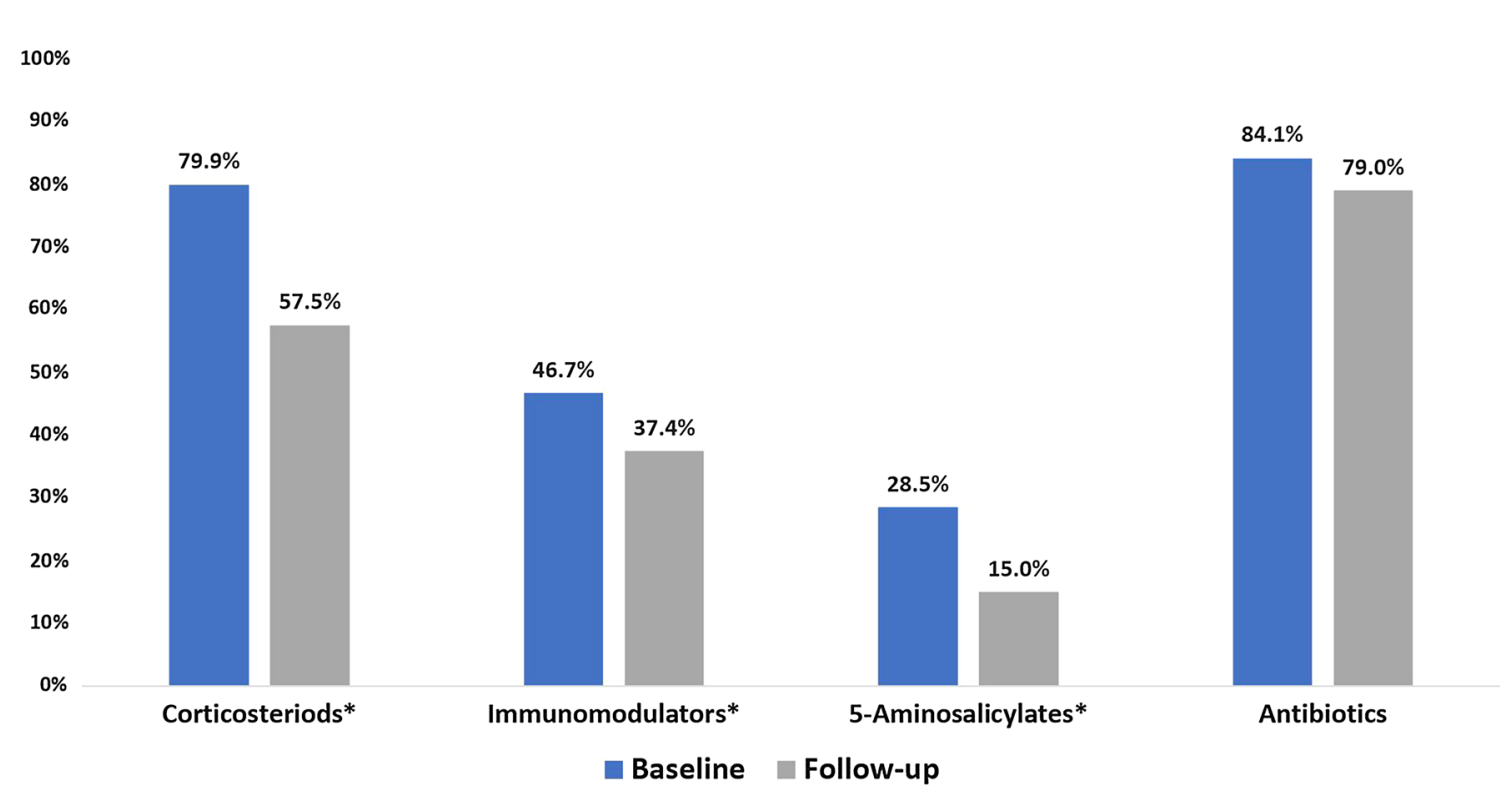

Fig. 2 Baseline versus follow-up analysis of $\mathrm{CD}$ related medications. CD Crohn's disease; UST ustekinumab. between the pre- and post-index period use of corticos${ }^{*}$ There was a significant difference $(p$ value $<0.05)$

\section{teroids, immunomodulators, and 5-aminosalicylates}

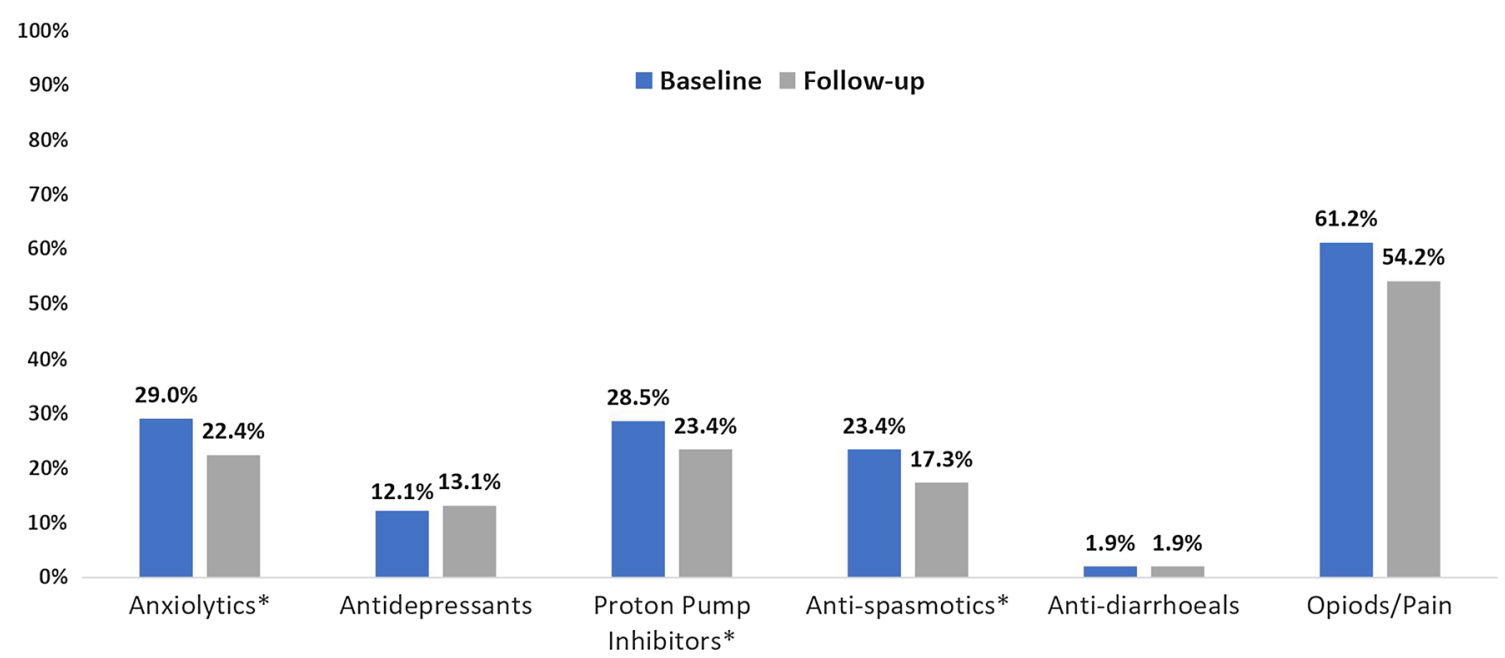

Fig. 3 Baseline versus follow-up analysis of medications for CD-related symptoms or comorbid conditions. $C D$ Crohn's disease; UST ustekinumab. ${ }^{*}$ There was a

significant difference $(p$ value $<0.05)$ between the preand post-index period use of anxiolytics, proton pump inhibitors, and anti-spasmodics

patients continued their initial biologic (ADA, CZP, golimumab, IFX, VDZ) treatment 1 year after initiation [34].

The present UST study found that most CD patients were persistent, and $83.6 \%$ remained persistent 1 year after initiation of UST. The average duration of treatment was 329 days. According to the FDA label for UST to treat moderately to severely active $\mathrm{CD}$, a subcutaneous (SQ) dose of UST should be used every 8 weeks after the initial IV dose [35]. However, in this study, $78.0 \%$ patients had subcutaneous UST for their index claim. It is possible that, in the real world, patients either skipped their IV dose and started subcutaneous injection, or the study did not capture the first actual claim for 
Table 4 Pre-post analysis of health care resource utilization and associated costs (in US\$)

\begin{tabular}{|c|c|c|c|c|c|}
\hline & \multicolumn{2}{|c|}{ Baseline period } & \multicolumn{3}{|c|}{ Follow-up period } \\
\hline & $n /$ Mean & $\% / S D$ & $n /$ Mean & $\% / S D$ & $p$ value \\
\hline \multicolumn{6}{|c|}{ All-cause health care resource utilization } \\
\hline Any ER visit & 102 & $47.7 \%$ & 81 & $37.9 \%$ & 0.013 \\
\hline Number of ER visits & 1.2 & 2.3 & 1.1 & 3 & 0.405 \\
\hline Any inpatient stay & 89 & $41.6 \%$ & 62 & $29.0 \%$ & 0.004 \\
\hline Number of inpatient stays & 0.7 & 1 & 0.5 & 1.1 & 0.083 \\
\hline Length of inpatient stay, days & 8.1 & 8.8 & 8.8 & 12.7 & 0.233 \\
\hline \multicolumn{6}{|c|}{ CD-related health care resource utilization } \\
\hline Any ER visit & 74 & $34.6 \%$ & 42 & $19.6 \%$ & $<0.001$ \\
\hline Number of ER visits & 0.6 & 1.4 & 0.4 & 1.6 & 0.018 \\
\hline Any inpatient stay & 87 & $40.7 \%$ & 60 & $28.0 \%$ & 0.004 \\
\hline Number of inpatient stays & 0.6 & 0.9 & 0.5 & 1 & 0.077 \\
\hline Any CD Surgery & 148 & $69.2 \%$ & 134 & $62.6 \%$ & 0.144 \\
\hline$\#$ of any CD Surgeries & 1.4 & 1.8 & 1 & 1.1 & 0.010 \\
\hline Length of inpatient stay, days & 7.5 & 8.6 & 8.4 & 11.4 & 0.246 \\
\hline \multicolumn{6}{|l|}{ All-cause health care costs } \\
\hline ER cost & $\$ 2182$ & $\$ 6132$ & $\$ 1373$ & $\$ 3752$ & 0.013 \\
\hline Inpatient costs & $\$ 14,054$ & $\$ 28,136$ & $\$ 12,368$ & $\$ 30,846$ & 0.500 \\
\hline \multicolumn{6}{|l|}{ CD-related health care costs } \\
\hline ER cost & $\$ 1293$ & $\$ 4527$ & $\$ 478$ & $\$ 1824$ & 0.002 \\
\hline Inpatient cost & $\$ 12,701$ & $\$ 27,078$ & $\$ 10,873$ & $\$ 28,598$ & 0.457 \\
\hline CD Surgery costs & $\$ 6534$ & $\$ 13,920$ & $\$ 6606$ & $\$ 21,906$ & 0.967 \\
\hline
\end{tabular}

$p$ values $<0.05$ were considered significant

$C D$ Crohn's disease; $S D$ standard deviation; UST ustekinumab

the IV injection due to lack of permanent J codes for ustekinumab for the majority of the study period. Among patients who discontinued treatment, $65.7 \%$ did not use any biologics after discontinuation and $26 \%$ restarted UST during the follow-up period. The majority of the patients who initiated treatment with UST were biologic experienced and had failed therapy with 1 or 2 biologics (ADA, CZP, IFX, or VDZ). Further studies comparing UST to other biologics are needed to assess persistence rates over more extended follow-up periods. The most common reasons for discontinuation of biologic treatment among CD patients include disease progression, loss of response, and infection events [34]. The high real-world persistence we found in the current study suggests effectiveness of UST 1 year after initiation. The results warrant future real-world studies of longer-term persistence.

Patients may lose their response to biologics over time, necessitating dose titration [18]. Rubin et al. [18] noted that dose escalation (dose doubling in the case of biologics) occurred 
among $16.7 \%$ of ADA users and $30.3 \%$ of IFX users in a real-world study. Einarson et al. [36] also noted that dose titration varied by line of treatment, with first line of treatment having the lowest rates of titration.

In this study, $77 \%$ of CD patients initiating treatment with UST used the FDA recommended doses (72-108 $\mathrm{mg} / 8$ weeks, within a $20 \%$ variation), $17.9 \%$ of patients had dose escalation, and $5.1 \%$ of patients had dose reduction. Similarly, another retrospective cohort study conducted in a large metropolitan IBD center reported that $17 \%$ of CD patients who initiated UST required dose intensification [37]. Notably, the dose escalation rate for UST is lower than that reported for biologics in other studies [18]. While it is possible that the definitions of dose titration vary across studies, and that this could influence the percentage of patients defined as undergoing titration, the results of the current study do show a promising real-world association between UST and low rates of dose escalation, which warrants further comparative studies. There are a few possible reasons for the dose reduction results reported. First, the average dose was calculated over 12 months in a mixed-population sample that consisted of $\sim 25 \%$ bio-naïve patients, thus, it is possible that the dose reductions occurred in this subset of patients. Secondly, the average dose was calculated from actual claims filled or delivered to patients, so may reflect delays in filling of claims or nonadherence and not necessarily clinician-prescribed dose reductions. Thirdly, it is also possible that there could be subsequent delays in filling of claims for insurer coverage reasons (though this should be less likely after initial SQ fills, since payer approvals for maintenance coverage would most likely be obtained prior to the initial SQ claim). Future studies using electronic medical records to examine the physician prescription order can help inform dose titration rates intended by physicians and further help verify the results of this claims-based study. Another interesting observation from the current study is that the Optum database (38.8\%) had more bio-naïve patients as compared with the IBM $(17.6 \%)$ and IQVIA (20.5\%) databases. Optum (12.8\%) also had a lower proportion of patients who required dose escalation as compared with IBM (20.7\%) and IQVIA (19.7\%), suggesting a possible relationship between biologic naïveté and treatment pattern outcomes.

Reductions in pre-/post-immunomodulators use have previously been reported in prior studies with other biologics among CD patients [38]. This study also observed a reduction in the use of corticosteroids, immunomodulators, and 5-aminosalicylates, anxiolytics, and antispasmodics during the 12 months post-initiation of UST. This suggests that UST use is associated with reductions in the use of concomitant immunomodulators, but it must be noted that the reduction in immunomodulator use may also be attributable to recent shifts in clinical decision around use of combination therapy. Further research is needed to better isolate the response to UST therapy with and without the use of concomitant immunomodulators.

The hospitalization rate in this study, calculated over a period of 12 months during the follow-up, was $29 \%$ for the CD patients treated with UST. By comparison, descriptive hospitalization rates among $\mathrm{CD}$ patients treated with biologics in other studies ranged from $16.7 \%$ for ADA to $30.4 \%$ for CZP [18]. The increased utilization of CD-related medications at baseline, the high percentage of bio-experienced patients at baseline, and the increased HCRU in our study are consistent with a population with potentially high disease severity. However, our study included no objective assessments of CD disease severity in order to examine its impact on treatment outcomes. Future studies using data in which objective clinical assessments are available may help in understanding the impact of baseline disease severity on response to therapy.

The present study also demonstrated a reduction in ER visits, inpatient stays, and CDrelated surgeries from the 12-month pre-index compared to the 12-month follow-up period. Average all-cause and CD-related costs for ER visits reduced significantly among patients treated with UST during the post-index period compared to the pre-index period. Inpatient stay costs also showed a numeric reduction after initiating UST, but this was not statistically significant. Compared to CD patients who 
responded to UST, CD patients with suboptimal or no response to UST had higher ER visit costs, inpatient service-related costs, and total medical costs [17]. These findings suggest that response to UST may be associated with decreased HCRU.

As with all retrospective, observational analysis, this study was limited to determining associations, and causality cannot be inferred. In addition, administrative claims data may contain coding errors and missing information and are not clinically comprehensive. This study also had unique limitations. As UST was first approved for the treatment of CD by the FDA in 2016, the sample size was limited, and analysis had an identification period that began in 2017. The high proportion of UST treated patients with prior biologic experience is not surprising given the recent FDA approval. This suggests the sample may be biased towards having more complicated or higher severity CD but could not be assessed with certainty in the present study due to lack of objective clinical measures of disease severity. Hence, data on clinical and economic outcomes should be interpreted with caution.

The study relied on HCPCS codes and NDC to identify UST claims. However, permanent HCPCS codes for UST IV dose were not available for most of the identification period in this study. Therefore, the study relied on HCPCS and NDC codes whenever possible to identify the first induction IV dose for UST. The study also included patients who started on SQ UST as their first clearly identifiable UST claim. It is possible that some patients may have received an IV dose for UST, whom we were unable to clearly identify in the claims data due to the lack of permanent HCPCS codes for IV induction dose. However, it is also possible that patients were started on SQ UST instead of the IV induction recommended in the FDA labeling for $\mathrm{CD}$. Hence, these results should be interpreted with this limitation in mind, and they warrant future research to examine ustekinumab initiation after January 1, 2018, when the permanent J code for IV ustekinumab became available. This study also did not incorporate complete public insurance databases (i.e., Medicare or Medicaid), hence the findings may not be generalizable to the entire population of the United States. Moreover, prescription claim dates might not have indicated the actual date of drug intake, or reasons for discontinuation or switch to another medication. Additionally, dose titration could not be directly evaluated in a clinical setting and was instead inferred from prescription claims. Recent FDA approval also limited examination of dose titration outcomes, which would be more comprehensive with longer follow-up, given the long treatment schedules of $\mathrm{CD}$ patients; since UST is dosed once every 8 weeks according to the US label, only an average of 6 maintenance doses would be administered within a 12-month follow-up period. Moreover, CD diagnosis and baseline comorbidities were identified from claims data using ICD-9/10 codes and not based on actual laboratory values or clinical assessment. Claims databases are designed for reimbursement purposes and are subject to potential coding mistakes and inaccuracies. Thus, we were unable to identify the exact location of the disease or disease severity based on objective measures of clinical assessment.

\section{CONCLUSIONS}

This study examined the real-world treatment and utilization patterns of UST among patients with $\mathrm{CD}$ in a large, pooled commercial database. High rates of persistence and low rates of dose escalation were found among CD patients treated with UST in the 12-month follow-up period. Reductions in steroid and some other medication use as well as health care resource utilization and associated costs were also noted after UST treatment initiation. The results warrant further studies that assess the effectiveness of UST over longer follow-up periods, and compare to other biologics used to treat CD.

\section{ACKNOWLEDGEMENTS}

Funding. This study and its submission for publication, including the journal's rapid 
service and open access fee, was funded without restriction by Janssen Scientific Affairs, LLC, 920 Rte. 202, Raritan, NJ 08869. All authors had full access to all data in this study and take complete responsibility for the integrity of the data and accuracy of the data analysis.

Medical Writing and Editorial Assistance. Editorial assistance in the preparation of this article was provided by Michael Kane of STATinMED Research. Medical writing support was provided by Janvi Sah of STATinMED Research and Hema Kannan Gandhi of SAV Consulting, LLC. Support for this assistance was funded by Janssen Scientific Affairs, LLC.

Authorship. All named authors meet the International Committee of Medical Journal Editors (ICMJE) criteria for authorship for this article, take responsibility for the integrity of the work as a whole, and have given their approval for this version to be published.

Disclosures. Camilo Obando, Zhijie Ding, and Erik Muser are currently employed by Janssen Scientific Affairs, LLC. Xiaoxi Sun, Huiqi Wang, Rajesh Mallampati, and Lin Xie are paid employees of STATinMED Research, which is a paid consultant to Janssen Scientific Affairs, LLC. Wenqin Qiang and Neel Vaidya were paid employees of STATinMED Research (a paid consultant to Janssen Scientific Affairs, LLC) at the time of the study. This study was funded, without restriction, by Janssen Scientific Affairs, LLC, including all expenses and fees associated with publication.

Compliance with Ethics Guidelines. The core study did not involve the collection, use or transmittal of individual identifiable data, and instead relied solely on the secondary use of depersonalized data; hence, review board approval was not required. Secondary use of depersonalized data is explicitly exempted from ethics review per the "Common Rule": 45 CFR 46; Exemption 4); Federal Policy for the Protection of Human Subjects (1991), and depersonalized data itself is not covered by the Privacy Rule, 45 CFR $\S 164.514$ (b)(2)(i); Health Insurance Portability and Accountability Act of
1996 (HIPAA). Nonetheless, the security of the data still meets HIPAA requirements, and by default, the study conformed with the Helsinki Declaration of 1964, as revised in 2013, concerning human and animal rights, and Springer's policy concerning informed consent.

Data Availability. Data sharing is not applicable to this article as no datasets were generated or analyzed during the current study.

Open Access. This article is licensed under a Creative Commons Attribution-NonCommercial 4.0 International License, which permits any non-commercial use, sharing, adaptation, distribution and reproduction in any medium or format, as long as you give appropriate credit to the original author(s) and the source, provide a link to the Creative Commons licence, and indicate if changes were made. The images or other third party material in this article are included in the article's Creative Commons licence, unless indicated otherwise in a credit line to the material. If material is not included in the article's Creative Commons licence and your intended use is not permitted by statutory regulation or exceeds the permitted use, you will need to obtain permission directly from the copyright holder. To view a copy of this licence, visit http:// creativecommons.org/licenses/by-nc/4.0/.

\section{REFERENCES}

1. Lichtenstein GR, Loftus EV, Isaacs KL, Regueiro MD, Gerson LB, Sands BE. ACG clinical guideline: management of Crohn's disease in adults. Am J Gastroenterol. 2018;113:481-517.

2. Louis E, Collard A, Oger AF, et al. Behaviour of Crohns disease according to the Vienna classification: changing pattern over the course of the disease. Gut. 2001;49(6):777-82. https://doi.org/10. 1136/gut.49.6.777.

3. Feuerstein JD, Cheifetz A. Crohn disease: epidemiology, diagnosis and management. Mayo Clin Proc. 2017;92(7):1088-103.

4. Crohn's \& Colitis Foundation of America (CCFA). The facts about inflammatory bowel diseases. 2014. 
http://www.crohnscolitisfoundation.org/assets/ pdfs/updatedibdfactbook.pdf. Accessed 9 Aug 2018.

5. Nguyen GC, Chong CA, Chong RY. National estimates of the burden of inflammatory bowel disease among racial and ethnic groups in the United States. J Crohns Colitis. 2014; 2014:288-295.

6. Cohen RD. The quality of life in patients with Crohn's disease. Aliment Pharmacol Ther. 2002;16(9):1603-9.

7. Kappelman MD, Porter CQ, Galanko JA, Rifas-Shiman SL, Ollendorf DA, Sandler RS, Finkelstein JA. Utilization of healthcare resources by US children and adults with inflammatory bowel disease. Inflamm Bowel Dis. 2011; 17(1):62-8.

8. Peery AF, Dellon ES, Lund J, et al. Burden of gastrointestinal disease in the United States: 2012 update. Gastroenterology. 2012;143(5). https://doi. org/10.1053/j.gastro.2012.08.002.

9. Hanauer S, Sandborn W. Management of Crohns disease in adults. Am J Gastroenterol. 2001;96(3): 635-43. 9270(01)02234-1.

10. Hilsden R. Funding the new biologics—what can we learn from infliximab? The CCOHTA report: a gastroenterologist's viewpoint. Can J Gastroenterol. 2002;16(12):865-8.

11. Targan SR, Feagan BG, Fedorak RN, Lashner BA, Panaccione R, Present DH, et al. Natalizumab for the treatment of active Crohn's disease: Results of the ENCORE trial. Gastroenterology. 2007;132(5): 1672-83. https://doi.org/10.1053/j.gastro.2007.03. 024 .

12. Brady J, Stott-Miller M, Mu G, Perera S. Treatment patterns and sequencing in patients with inflammatory bowel disease. Clin Ther. 2018;40(9): 1509-21.

13. Lelli F, Nuhoho S, Lee XY, Xu W. Systematic review: treatment pattern and clinical effectiveness and safety of pharmaceutical therapies for Crohn's disease in Europe. Clin Exp Gastroenterol. 2016;9: 311-23.

14. Gisbert J, Marin A, McNicholl A, Chaparro M. Systematic review with meta-analysis: the efficacy of a second anti-TNF in patients with inflammatory bowel disease whose previous anti-TNF treatment has failed. Aliment Pharmacol Ther. 2015;41(7): 613-23.

15. Gordon N, Sebastian S. Real-world success of biologic therapy in IBD: no more reasons to be anti antibody. Dig Dis Sci. 2019;64(3):614-5.
16. Baert F, Noman M, Vermeire S, et al. Influence of immunogenicity on the long-term efficacy of infliximab in Crohn's disease. $\mathrm{N}$ Engl J Med. 2003;348(7):601-8.

17. Moss A. Optimizing the use of biological therapy in patients with inflammatory bowel disease. Gastroenterol Rep. 2015;3(1):63-8.

18. Rubin D, Mody R, Davis K, Wang C. Real-world assessment of therapy changes, suboptimal treatment and associated costs in patients with ulcerative colitis or Crohn's disease. Aliment Pharmacol Ther. 2014;39(10):1143-55.

19. Rubin D, Uluscu O, Sederman R. Response to biologic therapy in Crohn's disease is improved with early treatment: an analysis of health claims data. Inflamm Bowel Dis. 2012;18(12):2225-31.

20. Ben-Horin S, Chowers Y. Review article: loss of response to anti-TNF treatments in Crohn's disease. Aliment Pharmacol Ther. 2011;33(9):987-95.

21. Benson JM, Peritt D, Scallon BJ, et al. Discovery and mechanism of ustekinumab: a human monoclonal antibody targeting interleukin-12 and interleukin23 for treatment of immune-mediated disorders. MAbs. 2011;3(6):535-45.

22. Sandborn WJ, Feagan BG, Gasink C, et al. OC-057 A PH3 Randomised, multicenter, double blind, placebo (PBO)-controlled study of ustekinumab (UST) maintenance therapy in moderate-severe Crohn's disease (CD) PTS: Results from IM-UNITI. Gut. 2016;65(Suppl. 1):A32.2-A34.

23. Sandborn WJ, Sands BE, Gasink C, et al. Sa1743Reduced rates of-Crohn's-related surgeries, hospitalizations and alternate biologic initiation with ustekinumab in the IM-UNITI study through 2 years. Gastroenterology. 2018;154(6, Suppl 1): S377-8.

24. Broder MS, Neary MP, Chang E, Cherepanov D, Katznelson L. Treatments, complications, and healthcare utilization associated with acromegaly: a study in two large United States databases. Pituitary. 2014;17(4):333-41.

25. Schepman PA, Knight T, Bond C, et al. Medical resource use in dermatomyositis/polymyositis patients treated with adrenocorticotropic hormone. In: Presented at the 8th Annual Perspectives in Rheumatic Diseases Conference, Las Vegas, NV, September 18-19, 2015.

26. Broder MS, Neary MP, Chang E, Katznelson L. Treatments, complications, and healthcare utilization associated with acromegaly: a study in two large United States databases. Pituitary. 2014;17: 333-41. 
27. Bernstein CN, Blanchard JF, Rawsthorne P, Wajda A. Epidemiology of Crohn's disease and ulcerative colitis in a central Canadian province: a population-based study. Am J Epidemiol. 1999;149(10): 916-24.

28. Shaw SY, Blanchard JF, Bernstein CN. Association between the use of antibiotics and new diagnoses of Crohn's disease and ulcerative colitis. Am J Gastroenterol. 2011;106(12):2133-42.

29. Yantiss RK, Odze RD. Diagnositc difficulties in inflammatory bowel disease pathology. Histopathology. 2006;48(2):116-32.

30. Geboes K, Colombel JF, Greenstein A, et al. Inderminate colitis: a review of the concept-what's in a name? Inflamm Bowel Dis. 2008;14(6):850-7.

31. Patel H, Lissoos T, Rubin DT. Indicators of suboptimal biologic therapy over time in patients with ulcerative colitis and Crohn's disease in the United States. PLoS ONE. 2017;12(4):e0175099.

32. Triantafillidis JK, Merikas E, Georgopoulos F. Current and emerging drugs for the treatment of inflammatory bowel disease. Drug Des Devel Ther. 2011;5:185-210.
33. San Román AL, Muñoz F. Comorbidity in inflammatory bowel disease. World J Gastroenterol. 2011;17(22):2723-33.

34. Chen C, Hartzema AG, Xiao H, et al. Real-world pattern of biologic use in patients with inflammatory bowel disease: treatment persistence, switching, and importance of concurrent immunosuppressive therapy. Inflamm Bowel Dis. 2019;25(8):1417-27.

35. Accessdata.fda.gov. [online]. 2020. https://www. accessdata.fda.gov/drugsatfda_docs/label/2016/ 761044lbl.pdf. Accessed 29 Jan 2020.

36. Einarson TR, Bereza BG, Lee XY, Lelli F. Dose escalation of biologics in Crohn's disease: critical review of observational studies. Curr Med Res Opin. 2017;33(8):1433-49.

37. Young A, Tsistrakis S, Rubinov J, Kohan E, Hirten R, Ungaro R, Oikonomou I, et al. Ustekinumab dose intensification can be effective in Crohn's disease patients not responding to induction. Am J Gastroenterol. 2018;113:S351-2.

38. Obando C, Xie L, Vaidya N, Sah J. Evaluate the treatment patterns and healthcare utilizations for biologic therapies for Crohn's disease. In: Digestive disease week. Washington, DC: June 2-5, 2018. 